\title{
Balanced line for a 3-colored point set in the plane
}

\author{
Sergey Bereg* $\quad$ Mikio Kano ${ }^{\dagger}$ \\ Submitted: Aug 23, 2011; Accepted: Jan 23, 2012; Published: Feb 7, 2012 \\ Mathematics Subject Classification: 05C88
}

\begin{abstract}
In this note we prove the following theorem. For any three sets of points in the plane, each of $n \geq 2$ points such that any three points (from the union of three sets) are not collinear and the convex hull of $3 n$ points is monochromatic, there exists an integer $k \in\{1,2, \ldots, n-1\}$ and an open half-plane containing exactly $k$ points from each set.
\end{abstract}

\section{Introduction}

Bisecting two finite sets of points in the plane by a line is a simple exercise. The existence of such a line follows from the discrete version of the classical ham-sandwich theorem [2] that states that, for any $d$ finite point sets $S_{1}, S_{2}, \ldots, S_{d}$ in $\mathbb{R}^{d}$, there exists a hyperplane $h$ such that each open half-space bounded by $h$ contains at most half of points of each set $S_{i}$.

A short survey related to this paper is found in [1]. Another variation of the problem is about balanced lines [3, 4]. A set of points in the plane is in general position if any three points are not collinear. Given a set of $n$ black and $n$ white points in general position in the plane, a line $l$ is said to be balanced if each open half-plane bounded by $l$ contains precisely the same number of black points as white points. Our definition of balanced line is slightly different from [3] since we do require the line to pass through two points of the sets. Pach and Pinchasi [3] proved that the number of balanced lines is at least $n$ answering the question of George Baloglou.

Sharir and Welzl [4] found that balanced lines in the plane are related to halving triangles in $\mathbb{R}^{3}$. Let $P$ be a set of $2 n+1$ points in $\mathbb{R}^{3}$ in general position, i.e. no four points are coplanar. A halving triangle of $P$ is a triangle spanned by three points in $P$ such that the plane containing the three points bisects the remaining points of $P$ (i.e. an

*Department of Computer Science, University of Texas at Dallas, Box 830688, Richardson, TX 75083 USA. E-mail: besp@utdallas.edu

${ }^{\dagger}$ Department of Computer and Information Sciences, Ibaraki University, Hitachi, Ibaraki 316-8511, Japan. E-mail: kano@mx.ibaraki.ac.jp 
open half-space bounded by the plane contains exactly $n-1$ points of $P$ ). They proved that the number of halving triangles is at least $n^{2}$. This bound is tight since points in convex position have exactly $n^{2}$ halving triangles.

In this note we study balanced lines for three point sets. Let $S=R \cup B \cup G$ be a set of $3 n$ points in the plane in general position such that $|R|=|B|=|G|=n \geq 2$ (red, blue and green points). A line $l$ is called balanced if an open half-plane bounded by $l$ contains exactly $k$ red, $k$ blue and $k$ green points for some $k \in\{1,2, \ldots, n-1\}$. Unfortunately, a balanced line does not always exist, see an example in Figure 1 (b). To develop an intuition we check points on the line first.

It is known that if $n$ red points and $n$ blue points lie on a line in general position (i.e., no two points lie on the same position) and if the two end points have the same color, then there exists a balanced point.

Proposition 1 Assume that $n$ red points and $n$ blue points are given on the line and no two points lie on the same position, where $n$ is a positive integer. If both endpoints are red, then the line can be divided into two parts, the right part $I_{1}$ and the left part $I_{2}$, by a point so that $I_{1}$ contains $k$ red points and $k$ blue points for some $1 \leq k \leq n-1$.

Remark. Notice that the condition of Proposition 1 that both endpoints are the same color is necessary. For example, a configuration rrrbrbrbbb, where $r$ and $b$ denote a red point and a blue point, respectively, has no balanced point given in Proposition 1.

We will prove that a balanced line for points in the plane exists if the convex hull of $S$ is monochromatic.

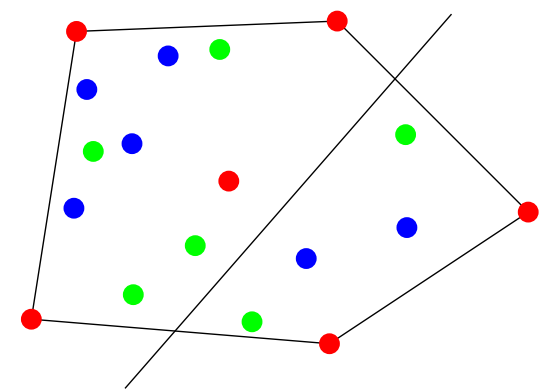

(a)

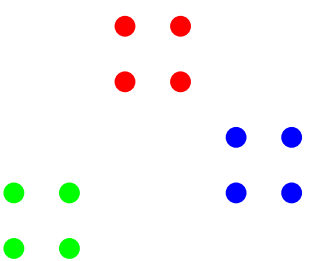

(b)

Figure 1: (a) Balanced line in a set of 18 points such that the convex hull is monochromatic. (b) A set of 12 points with non-monochromatic convex hull such that a balanced line does not exist.

Theorem 2 Let $S$ be a set of $3 n \geq 6$ points in the plane in general position colored in red/blue/green such that

(i) the number of points of each color is $n$, and

(ii) the vertices of the convex hull have the same color.

Then there exists a balanced line of $S$. 


\section{Existence of a Balanced Line}

In this Section we prove Theorem 2.

Proof. Let $d$ be a direction such that any two points of $S$ have different projections on a line with slope $d$. Let $p_{1}, \ldots, p_{3 n}$ be the order of points in direction $d$. For every $k$, let $r_{k}, b_{k}, g_{k}$ be the number of red/blue/green points in $\left\{p_{1}, \ldots, p_{k}\right\}$, respectively. Consider point $q_{k}=\left(3 b_{k}-k, 3 g_{k}-k\right)$. Note that $q_{k} \neq(0,0)$ if $k$ is not multiple of 3 . The theorem follows if $q_{k}=(0,0)$ for some $k=3,6, \ldots, 3(n-1)$. Suppose to the contrary that $q_{k} \neq(0,0)$ for any $k$ and any direction $d$.

Consider path $\phi_{d}=q_{1} q_{2} \ldots q_{3 n-1}$. By the definition $q_{1}=(-1,-1)$ and $q_{3 n-1}=(1,1)$, see Figure 2 (a). There are three types of vectors $\overrightarrow{q_{k-1} q_{k}}$ depending on the color of $p_{k}$, see Figure $2(\mathrm{~b})$. Note that the segments $q_{k-1} q_{k}$ do not contain grid points except the endpoints. Therefore path $\phi_{d}$ does not contain the origin. If we trace vector $\overrightarrow{0 a}$ where $a$ traverses path $\phi_{d}$ the turning angle of $a$, defined as $\sum_{i=1}^{3 n-2} \angle q_{i} O q_{i+1}$, will be $t \pi$ where $t$ is an odd integer.

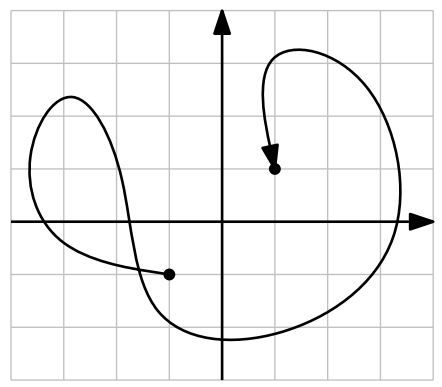

(a)

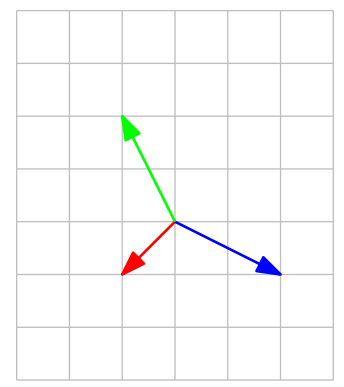

(b)

Figure 2: (a) Path $\phi_{d}$ with turning angle $\pi$. (b) Vectors $q_{k-1} q_{k}$ depending on the color of $p_{k}$.

We show that the turning angle of $\phi_{d}$ does not change with $d$. It suffices to consider a flip of two points $p_{k}$ and $p_{k+1}$ when $d$ changes. Suppose that $p_{k}$ is red and $p_{k+1}$ is blue. Then path $q_{k-1} q_{k} q_{k+1}$ changes to $q_{k-1} q_{k}^{\prime} q_{k+1}$ as shown in Figure 3 (a). We show that parallelogram $q_{k-1} q_{k} q_{k+1} q_{k}^{\prime}$ does not contain the origin. Suppose to the contrary that it contains the origin. Then $y\left(q_{k}\right)=0$ and $3 g_{k}=k$ and $k \equiv 0 \bmod 3$. On the other hand $x\left(q_{k}\right)=3 b_{k}-k \in\{-1,-2\}$ contradicting $k \equiv 0 \bmod 3$. The case, where $p_{k}$ is blue and $p_{k+1}$ is red, is symmetric.

Similarly, we can show that parallelogram $q_{k-1} q_{k} q_{k+1} q_{k}^{\prime}$ does not contain the origin if $p_{k}$ and $p_{k+1}$ have different colors, see Figure $2(\mathrm{~b})$ and (c). Note that $\phi_{-d}$ is symmetric to $\phi_{d}$ and its turning angle is $-t \pi$. This contradicts the fact that the turning angle $\phi_{d}$ does not change under rotation of $d$.

We finally note that the condition that the numbers of red, blue and green points are equal in Theorem 2 is also necessary. It is easy to make an example with distinct number 


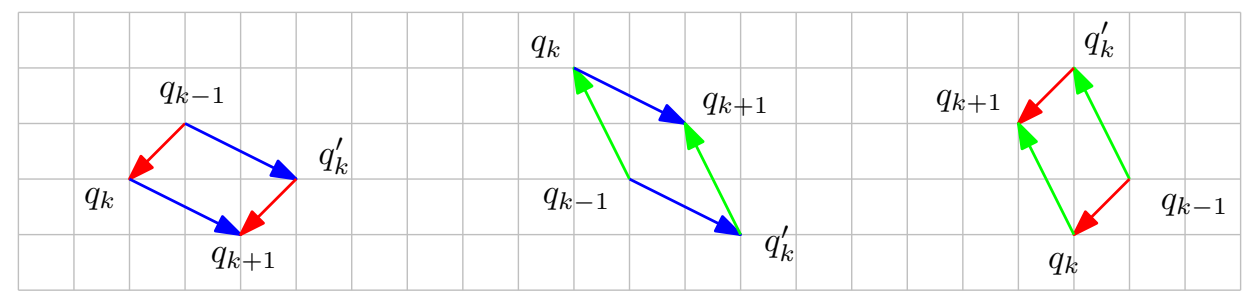

(a)

(b)

(c)

Figure 3: Flipping $p_{k}$ and $p_{k+1}$. Path $q_{k-1} q_{k} q_{k+1}$ changes to $q_{k-1} q_{k}^{\prime} q_{k+1}$. (a) $p_{k}$ is red and $p_{k+1}$ is blue. (b) $p_{k}$ is green and $p_{k+1}$ is blue. (c) $p_{k}$ is red and $p_{k+1}$ is green.

of points of each color that does not admit a balanced line. It is also natural to change the definition of balanced line in this case. For an red points, bn blue points and $c n$ green points are given in the plane in general position, a line $l$ is called balanced if an open half-plane bounded by $l$ contains exactly $a k$ red points and $b k$ blue points and $c k$ green points for some $k \in\{1,2, \ldots, n-1\}$. For example, the configuration of points shown in Figure 4 has no such balanced line.

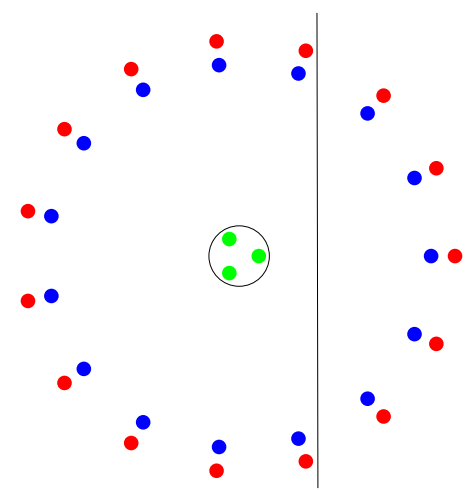

Figure 4: Example of 15 red, 15 blue and 3 green points without balanced line. Any line cutting off 5 red points does not intersect the circle enclosing green points.

\section{References}

[1] A. Kaneko and M. Kano. Discrete geometry on red and blue points in the plane A survey. In Discrete and Computational Geometry Algorithms and Combininatorics, pp. 551-570. Springer, 2003.

[2] J. Matoušek. Using the Borsuk-Ulam Theorem. Springer-Verlag, Heidelberg, 2003.

[3] J. Pach and R. Pinchasi. On the number of balanced lines. Discrete \& Computational Geometry, 25(4):611-628, 2001.

[4] M. Sharir and E. Welzl. Balanced lines, halving triangles, and the generalized lower bound theorem. In Symposium on Computational Geometry, pp. 315-318, 2001. 